\title{
CREATING AUDIENCE SPECIFIC GALACTIC SIMULATIONS USING EYE-TRACKING TECHNOLOGY
}

\author{
Janelle Arita \\ Department of Computer Science, Depauw University, 313 S. Locust Street, Greencastle, Indiana, USA \\ janellearita_2011@depauw.edu \\ Jenniffer Feliz \\ Department of Computer Science, Fordham University, 113 West 60th Street, New York City, New York, USA \\ jefeliz@fordham.edu \\ Dennis Rodriguez, Hans-Peter Bischof, Manjeet Rege, Reynold Bailey \\ Department of Computer Science, Rochester Institute of Technology, 1 Lomb Memorial Drive, Rochester, New York, USA \\ dxr4305@rit.edu,hpb@cs.rit.edu,mr@cs.rit.edu,rjb@cs.rit.edu
}

Keywords: Perception, eye-tracking, data visualization, galactic images.

\begin{abstract}
This study uses eye-tracking to investigate the differences in the way professional astrophysicists and novices observe simulations of galactic events. The results of this study provide insight into which aspects of the data are important and allow us to tailor the visualizations for a specific group. We hypothesized that the gaze patterns of professionals and novices would vary considerably. A user study was performed on two groups: trained astrophysicists and novices. Each group was presented with a randomized sequence of images and a video while their gaze patterns were recorded with an eye-tracker. We discovered that although both groups observed each image for the same duration, experts limited their fixations to a smaller area. Novices, on the other hand, had fixations which were spread across the images. For the video, the astrophysicists were more focused on simulations in which most of the data was visible and the camera angles had minimal axis change.
\end{abstract}

\section{INTRODUCTION}

Data visualization techniques allow us to better understand large multidimensional data and often reveal details that would generally be overlooked. The Center for Computational Relativity and Gravitation (CCRG) at Rochester Institute of Technology aims to further our understanding of astrophysical phenomena through the use of mathematical modeling, supercomputing and data visualization (Rochester Institute of Technology, 2010). The faculty at CCRG have developed an extensible and robust visualization system named Spiegel. Spiegel is highly versatile and capable of visualizing large amounts of multidimensional data, which allows for the analysis of the data in both time and space (Bischof, 2010). The Spiegel sytem has been shown to be useful for a wide range of visualization applications (Bischof et al., 2006) and several images generated by Spiegel have been featured in documentaries about black holes (A\&E Television Networks, 2010).

From a visualization perspective, it is crucial to understand whether the simulations created are scientifically meaningful and relevant to the professionals in the field. In this paper, we present an eye- tracking study that investigates the differences in the way that experts and novices look at galactic simulations. By extracting fixations from the eye-tracking data, we are able to determine the areas of interest in the data for each group. Understanding what aspects of the data the astrophysicists consider important allows for the creation of customized simulations which will be more relevant to professionals. The benefit of capturing eye movement data, as opposed to simply asking what viewers are paying attention to, is that people are often not aware of their own behavioral strategies (Eger et al., 2007). With eye-tracking data, visibility, meaningfulness, and placement can be objectively evaluated and the findings can be used to improve design (Goldberg and Kotval, 1999).

We hypothesized that experts would look at the simulations very differently than novices. It is probable that novices will focus more on visually appealing parts of the simulation or image, while experts will focus on areas that they find more important because of their advanced knowledge. In order to investigate this, our participants were presented with imagery from the Hubble Space Telescope (Space Telescope Science Institute, 2010) as well as images and a video generated by the Spiegel system. 


\section{RELATED WORK}

Eye-tracking systems first emerged in the early 1900s (Dodge and Cline, 1901). More recently, eyetracking has been used within a variety of contexts including aviation, driving, market research, psychology, and design (Goldberg and Kotval, 1999). Eyetrackers have also been used for human-computer interaction (Hutchinson et al., 1989), interactive graphics applications (Luebke et al., 2002; Levoy and Whitaker, 1990; O'Sullivan et al., 2003), and large scale display systems (Baudisch et al., 2003).

Researchers have investigated eye movements of novices and experts in different scenarios including surgeons performing aiming tasks on a laparoscopic surgery simulator (Law et al., 2004). A method has also been presented for improving visualization systems by using eye-tracking to determine what regions of the data viewers considered to be important ( $\mathrm{Lu}$ et al., 2010). Based on this information, reasonable rendering parameters were computed and the interactivity of the visualization system improved significantly. Grindinger and Duchowski automatically classified novice and expert pilots by analyzing their gaze patterns (Grindinger et al., 2010). The aim of our study is to build on the existing novice versus expert research, and to obtain the advantages that eyetracking can offer for improving data visualization.

\section{EXPERIMENTAL DESIGN}

\subsection{Apparatus}

A Mirametrix S1 eye-tracker operating at $60 \mathrm{~Hz}$ with gaze position accuracy less than 1 degree was used. The eye-tracker uses infrared illumination and an infrared camera to record video of the observer's eyes. The video is analyzed to locate the corneal reflection and pupil center and this information is used to determine the location on the screen where the observer is looking (Poole and Ball, 2005). Stimuli were presented on a 20 inch LCD monitor with a resolution of $1680 \times 1050$. Figure 1 shows a photograph of the experiment setup. The stimuli consisted of sixteen images (10 from Hubble Space Telescope, 6 from Spiegel) as well as a video generated using Spiegel. The images used are shown in Figure 2.

\subsection{Participants}

Twenty participants between the ages of 18 to 36 volunteered for this study. All participants were screened

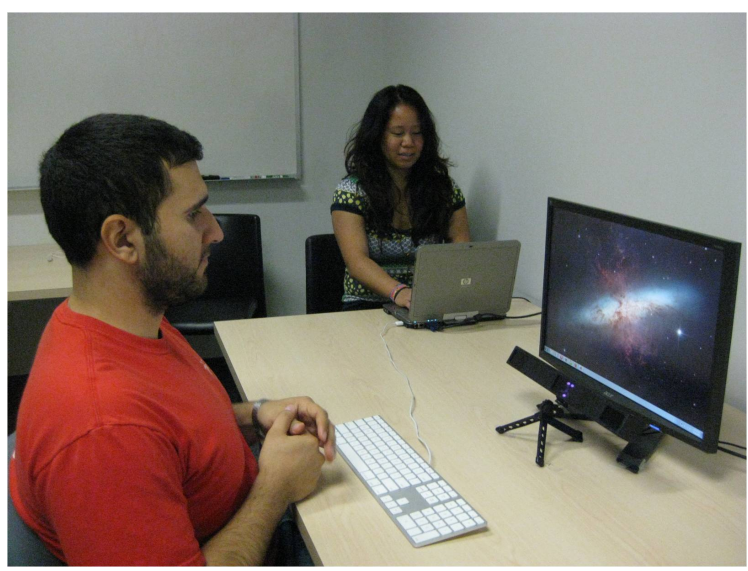

Figure 1: Experimental setup.

for impairments in visual acuity and color vision. Participants were separated into two groups - novices and experts, based on their knowledge of astrophysics. Sixteen participants were grouped into the novice category while 4 were grouped as experts.

- Expert group: Participants were selected for the expert group if they held at least a Masters degree in astrophysics. The selection of participants in the expert group was limited due to the criteria of knowledge on astrophysics. There were 4 male participants with an average age of 34 . Participants in this group were asked to rate their knowledge of astrophysics on a scale from 1 to 10 with 10 being the highest. The average rating was 7.25.

- Novice group: Participants were selected for the novice group if they had little or no knowledge about astrophysics. Knowledge was determined based on participants' prior courses and hobbies. There were 10 females, and 7 males with an average age of 21 . The average rating of knowledge on astrophysics for the novice group was 2.58 .

\subsection{Procedure}

The user study took place in a controlled room with moderate lighting. Each participant was seated approximately 20 inches in front of the screen and positioned such that both pupils were clearly identified by the eye-tracker. Participants were asked to minimize head movement during the experiment to ensure accurate eye tracking.

The participants were asked to view a randomized sequence of the sixteen images followed by the video. They were instructed to rate the quality of each image on a scale from 1 to 10 with 10 being the highest. Following each image, a blank screen was displayed for 3 seconds. While the blank screen was being displayed, the participants verbally expressed their rating 

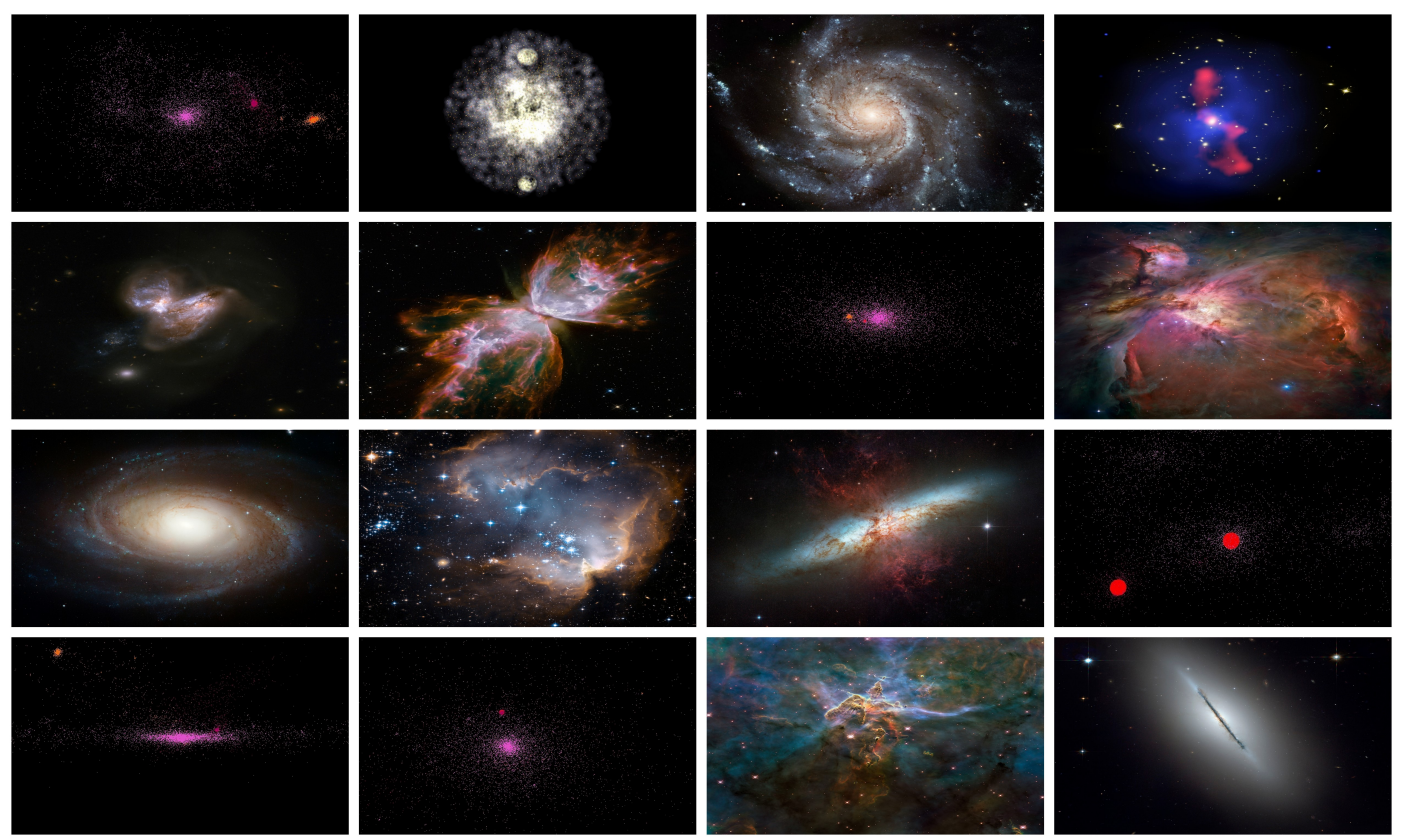

Figure 2: The 16 images that were shown as stimuli for the first phase of the study.

for the image they just viewed. The participants were not given a definition of quality. Instead it was left up to them to formulate their own notion of quality. The purpose of the ratings was to help the participant focus on the areas of the visuals which they considered most appealing. The complete set of instructions read verbatim to each participant can be found in Appendix A. Eye-tracking data collected from each participant was saved into an XML file. Each XML file contained the following data: time elapsed, location of fixation for both left and right eye, fixation duration, fixation ID, and file name of the image or video being displayed. Testing for each participant, including calibration, and vision and color tests, lasted approximately 10 minutes.

\section{RESULTS AND DISCUSSION}

\subsection{Images}

\subsubsection{Percentage of fixation duration}

To determine if the gaze patterns for the novices and experts were different, we divided each image into an $8 \times 8$ grid and compared the number of fixations in corresponding grid cells for the experts and novices. Figure 3 shows the average fixation distribution for the group of novices for one of the images in the test set. Figure 4 shows the average fixation distribution for the group of experts for the same image. Notice that the gaze pattern for the novices tends to be more spread out while the gaze pattern for the experts is more focused. A similar trend was observed for all of the images in the experiment. Although the gaze patterns for the experts tended to be more focused while the gaze patterns for the novices tended to be more spread out, there was still a strong correlation between the two groups. The Pearson coefficient of correlation between experts and novices for all sixteen images was $r=0.8763$. This suggests that both groups still spent the majority of time attending to the same salient features and likewise ignoring the same non-salient features. This supports the findings from previous studies which show that our focus is naturally drawn to regions of bright saturated colors, high edge density, high local contrast, and informative regions of an image (Mackworth and Morandi, 1967; Mannan et al., 1996; Parkhurst and Niebur, 2003).

\subsubsection{Number of Fixations}

The average number of fixations per image was also calculated for both groups (see Figure 5). More fixations per image may be an indicator or higher interest or focus or the result of a rapid visual search (Goldberg and Kotval, 1999). The number of fixations for the Hubble images tended to be higher than the number of fixations for the Spiegel images. This is 


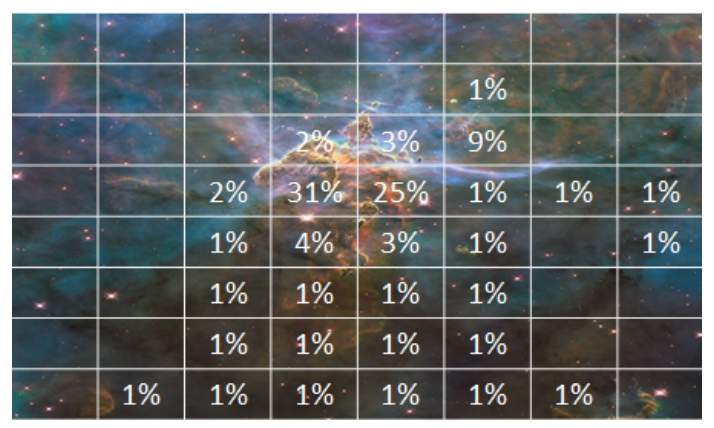

Figure 3: Average fixation time for novices for one image from the test set.

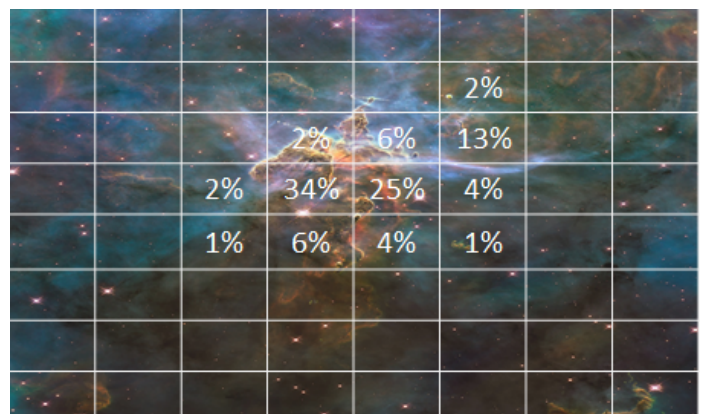

Figure 4: Average fixation time for experts for one image from the test set.

likely due to the fact that the Hubble photographs had richer content across the entire image compared to the Spiegel images. We also observed that there was a strong correlation between the number of fixations and the average quality rating. In particular, a larger number of fixations tended to result in a higher quality rating. The Pearson coefficient of correlation between image ratings and the number of fixations for experts was $r=0.7965$ and for novices it was $r=0.8750$.

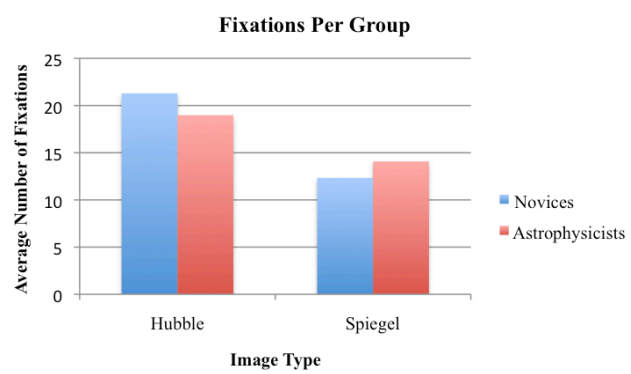

Figure 5: Average number of fixations for novices and experts.

\subsection{Video}

One of the problems that is often faced when creating visualizations is determining the best path that the camera takes through the data. Although very little work has been done on interpreting eye-tracking data of dynamic stimuli we believe that it holds great potential for aiding the design process. In the second phase of our study, we displayed a video to the participants. The video was divided into four quadrants, each showing a different flight path through the same data. The video in each quadrant was synchronized so that each event in the simulation occurred at the same time from different perspectives.

By displaying four flight paths at once, we determined which flight path was attended to most often by the two groups of participants. We determined this by calculating the fixation duration for each flight path for each group. The flight path that contained the most fixation duration was the flight path that was most observed. Using this approach, we determined that the experts spent more time looking at the flight path in the lower left quadrant, while the novices preferred the flight path in the top left. See Figures 6 and 7.

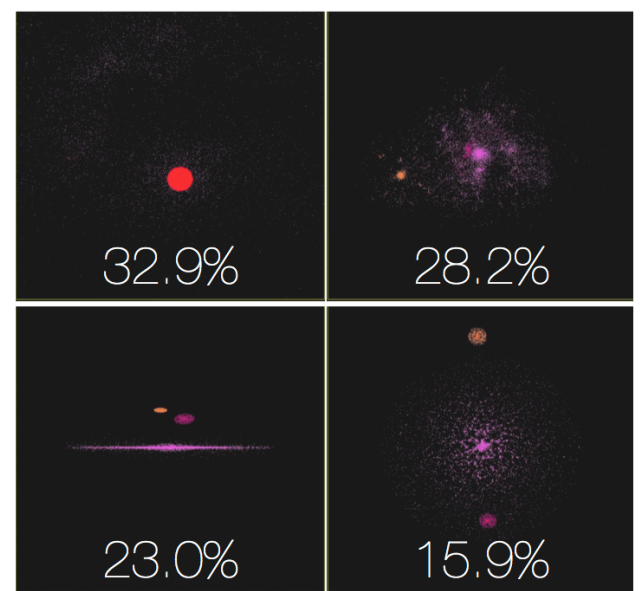

Figure 6: Percentage of fixation time spent in each quadrant of the video for the novices.

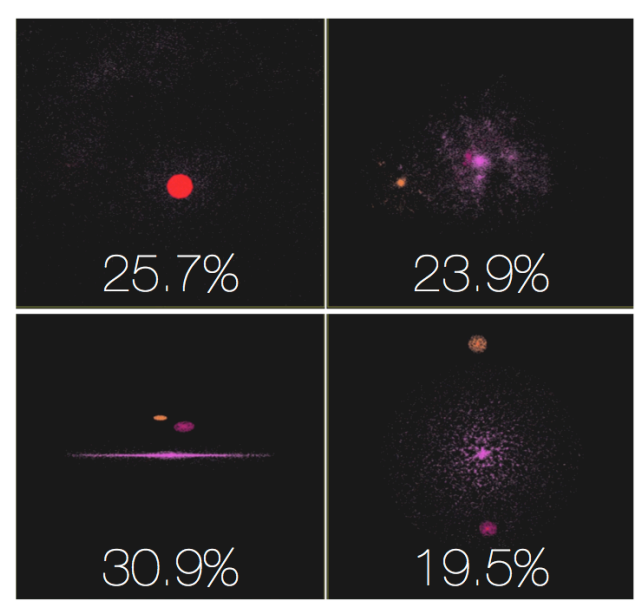

Figure 7: Percentage of fixation time spent in each quadrant of the video for the experts. 

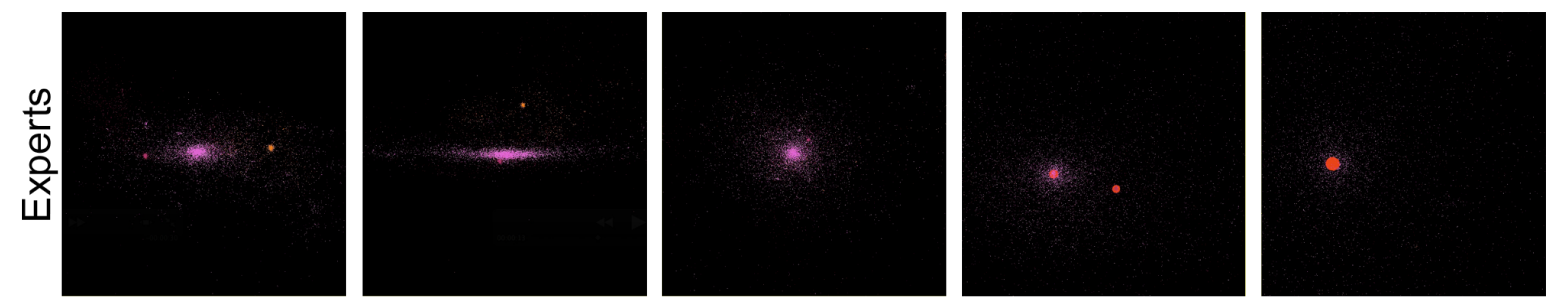

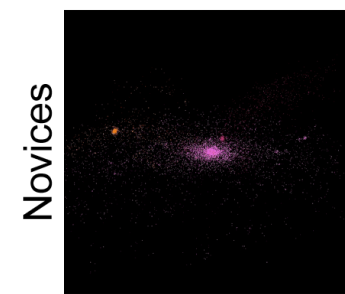

3

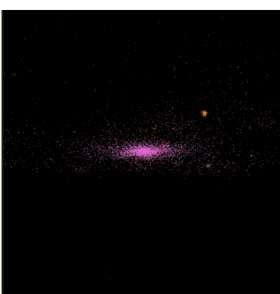

4

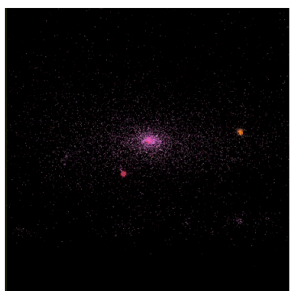

5

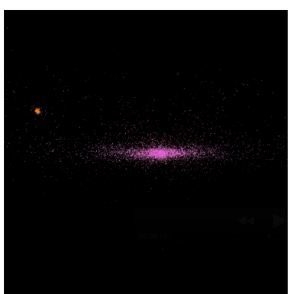

6

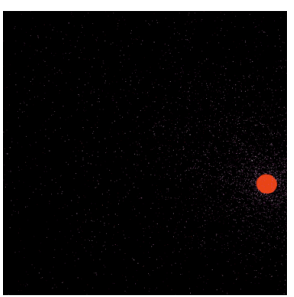

7

Figure 8: Screen shot of preferred flight path for segments 3 to 7.

This observation by itself is not very useful unless we take it one step further and analyze which of the four quadrants was attended to at specific stages of playback. To accomplish this, we divided the video into 14 segments, each segment spanning 3 seconds in length. By analyzing the video in smaller segments, we distinguished which flight path a participant fixated on at specific times. For example, Figure 8 shows a screen shot of the preferred flight path for segments 3 to 7 . We recreated an optimal audience specific video for each group by merging the preferred flight path segments into a single video.

\section{CONCLUSION AND FUTURE WORK}

We have presented an experiment which used eyetracking to compare the gaze patterns of novices and expert astrophysicists while looking at various images and simulations of galactic events. We noted that although experts and novices both focus on similar salient regions of an image and ignore similar non-salient regions that the novices' gaze tended to be more spread out while the experts' gaze tended to be restricted to fewer regions of the image. For the video, it was observed that the professional group was more focused on simulations in which most of the data was visible and the camera angles had minimal axis change. These results provide valuable insight that will be used for designing more relevant, audience specific visualizations.

Future work includes improvement of the simulation video by creating smoother camera transitions between segments. Instead of manually creating a simulation by merging segments, we can recreate the entire video by simulating the preferred camera flight path through the Spiegel simulation software. Ideally we would like to completely automate this process to allow the user to view several flight paths and have the system dynamically create the final simulation video based on the viewer's gaze preferences. In order to accomplish this, it will be necessary to interface the eye-tracker with the simulation software.

\section{REFERENCES}

A\&E Television Networks (2010). Cosmic holes: The History Channel. http://www.history.com/shows/ the-universe/episodes/season-2.

Baudisch, P., DeCarlo, D., Duchowski, A., and Geisler, W. (2003). Focusing on the essential: considering attention in display design. Commun. ACM, 46(3):60-66.

Bischof, H.-P. (2010). The Spiegel Project. http: //spiegel.cs.rit.edu/ hpb/grapecluster/ Spiegel/index.html.

Bischof, H.-P., Dale, E., and Peterson, T. (2006). Spiegel - a visualization framework for large and small scale systems. In In MSV 06: Proceedings of the 2006 International Conference of Modeling Simulation and Visualization Methods.

Dodge, R. and Cline, T. S. (1901). The angle velocity of eye movements. Psychological Review, 8:145-157.

Eger, N., Ball, L. J., Stevens, R., and Dodd, J. (2007). Cueing retrospective verbal reports in usability testing through eye-movement replay. In $\mathrm{BCS}-\mathrm{HCI}$ '07: Proceedings of the 21st British HCI Group Annual Conference on People and Computers, pages 129-137, Swinton, UK, UK. British Computer Society. 
Goldberg, J. H. and Kotval, X. P. (1999). Computer interface evaluation using eye movements: methods and constructs. International Journal of Industrial Ergonomics, 24(6):631 - 645 .

Grindinger, T., Duchowski, A. T., and Sawyer, M. (2010). Group-wise similarity and classification of aggregate scanpaths. In ETRA '10: Proceedings of the 2010 Symposium on Eye-Tracking Research \&\#38; Applications, pages 101-104, New York, NY, USA. ACM.

Hutchinson, T. E., White, K. P., Martin, W. N., Reichert, K. C., and Frey, L. A. (1989). Human-computer interaction using eye-gaze input. Systems, Man and $C y$ bernetics, IEEE Transactions on, 19(6):1527-1534.

Law, B., Atkins, M. S., Kirkpatrick, A. E., and Lomax, A. J. (2004). Eye gaze patterns differentiate novice and experts in a virtual laparoscopic surgery training environment. In ETRA '04: Proceedings of the 2004 symposium on Eye tracking research \& applications, pages 41-48, New York, NY, USA. ACM.

Levoy, M. and Whitaker, R. (1990). Gaze-directed volume rendering. SIGGRAPH Comput. Graph., 24(2):217223.

Lu, A., Maciejewski, R., and Ebert, D. S. (2010). Volume composition and evaluation using eye-tracking data. ACM Trans. Appl. Percept., 7(1):1-20.

Luebke, D., Watson, B., Cohen, J. D., Reddy, M., and Varshney, A. (2002). Level of Detail for 3D Graphics. Elsevier Science Inc., New York, NY, USA.

Mackworth, N. H. and Morandi, A. J. (1967). The gaze selects informative details within pictures. Perception and Psychophysics, 2:547-552.

Mannan, S. K., Ruddock, K. H., and Wooding, D. S. (1996). The relationship between the locations of spatial features and those of fixations made during visual examination of briefly presented images. Spatial Vision, 10:165-188.

O'Sullivan, C., Dingliana, J., and Howlett, S. (2003). Eyemovements and interactive graphics. The Mind's Eyes: Cognitive and Applied Aspects of Eye Movement Research, pages 555-571. J. Hyona, R. Radach, and H. Deubel (Eds.).

Parkhurst, D. and Niebur, E. (2003). Scene content selected by active vision. Spatial Vision, 16:125-154.

Poole, A. and Ball, L. J. (2005). Eye tracking in humancomputer interaction and usability research: Current status and future. In Prospects, Chapter in C. Ghaoui (Ed.): Encyclopedia of Human-Computer Interaction. Pennsylvania: Idea Group, Inc.

Rochester Institute of Technology (2010). Center for Computatonal Relativity and Gravitation. http://ccrg. rit.edu/.

Space Telescope Science Institute (2010). Hubblesite. http://hubblesite.org.

\section{APPENDIX}

\section{A. INSTRUCTIONS TO PARTICIPANTS}

In addition to the following instructions, which were read to the participants at the start of the study, participants were also given documentation showing that the study was reviewed and approved by the Institutional Review Board (IRB) at the institution where this study was conducted.

The purpose of this study is to gain a better understanding of how humans look at images of galactic events.

You will be shown a sequence of images and a video. Your task will be to evaluate the quality of each image and video by assigning a rating from 1 to 10 with 1 being the lowest quality and 10 being the highest. Please state your rating when the blank screen between images is displayed. The images that you will be viewing consist of several images from the Hubble Space Telescope as well as computer generated images. You will also see a computer generated video.

During the course of the experiment, a noninvasive camera will be used to record your eye movements. Please try to minimize your head movements as this may adversely affect the quality of the results. A short calibration process is necessary to ensure that your eyes are being accurately tracked. This will occur at the start of the experiment. Calibration simply involves looking at the targets on the screen until they disappear. The entire experiment should take no longer than $10 \mathrm{~min}$ utes to complete.

The results of this study may be published in scientific research journals or presented at professional conferences. However, your name and identity will not be revealed and your record will remain anonymous. Your name will not be used in any data collection, so it will be impossible to tell your answers from other peoples answers.

The potential benefits of this study to society include improvements in data visualization techniques and the advancement of scientific knowledge of human visual perception. Participation is entirely voluntary. Additionally, you may choose to withdraw from this study at any time. If you decide not to participate or to withdraw from this study, there will not be a penalty to you. Do you have any questions before we begin? 